\title{
Schizoneura gondwanensis FEISTMANTEL DA FORMAÇĀO RIO DO RASTO (BACIA DO PARANÁ, PERMIANO SUPERIOR) NO ESTADO DO PARANA E NO NORTE DO ESTADO DE SANTA CATARINA ${ }^{1,2}$
}

\author{
Rosemarie Rohn ${ }^{3}$
}

Oscar Rösler ${ }^{4}$

\begin{abstract}
Specimens of Schizoneura gondwanensis are here described from 13 new outcrops of the Rio do Rasto Formation (Passa Dois Group, Paraná Basin, Upper Permian) in Paraná State and northern Santa Catarina State. This species was previously known in Brazil from only two other localities of the same formation. The foliage shoots, preserved as impressions in red and gray mudstones, siltstones and fine sandstones, generally occur as fragments, which may, however, exhibit well-preserved details such as possible structures of the mesophyll and epidermis, only very rarely recognized in other Gondwana specimens of Schizoneura. The fossils also corroborate Feistmantel's observations made in 1880 , but overlooked by many later authors, regarding the embracing of the leaf clusters at the nodes, commissural lines, veins, etc. Although the leaf clusters at one outcrop are oblong-oval and not oval-lanceolate as at the other outcrops, all are tentatively considered as belonging to the same species. Several of the leaf clusters of $S$ gondwanensis present ruptures along the commissural lines, here interpreted as the result of taphonomic processes or, possibly, environmental factors that affected the living plant. Ontogenetic aspects of Schizoneura and comparisons with Cathaysian representatives of this genus are also discussed. $S$. gondwanensis thus has a much broader geographical range in Brazil than previously thought and, consequently, can now be considered as one more important element of Rösler's Taphoflora E.
\end{abstract}

\section{INTRODUÇĀO}

Fósseis do gênero Schizoneura Schimper \& Mougeot, 1844 caracterizam-se por estreitos caules articulados que, em cada nó, apresentam folhas lineares, soldadas total ou parcialmente entre si, constituindo, em geral, dois conjuntos foliares distintos e opostos. Este gênero está representado na Flora Glossopteris do Permiano e no Triássico do Gondvana, na Flora Gigantopteris do Permiano Superior da Cathaysia, no Permo-triássico de Angara e no Triássico e Jurássico da Europa. A espécie Schizoneura gondwanensis Feistmantel, 1876 ocorre em diversas regiōes gondvānicas, porém, no Brasil, doze exemplares seguramente atribuiveis a esta espécie, procedentes de apenas dois afloramentos da Formaçâo Rio do Rasto, foram anteriormente registrados (CAZZULO-KLEPZIG, 1978; CAZZULO-KLEPZIG \& CORREIA, 1981).

No presente trabalho, descreve-se $S$. gondwanensis com base em 51 exemplares de um total de 89 , coletados em 13 novas localidades da faixa de afloramentos da Formaçāo Rio do Rasto (Grupo Passa Dois, Permiano Superior) do Estado do Paraná e do norte de Santa Catarina. Embora relativamente fragmentados, alguns exemplares permitem o reconhecimento de importantes particularidades que confirmam as observações apresentadas por apenas poucos outros autores (FEISTMANTEL, 1880; PANT et al., 1982).

Este trabalho é parte de uma pesquisa mais ampla sobre a bioestratigrafia e paleoambiente da Formação Rio do Rasto, em relação à qual já estão concluídos os trabalhos referentes

1 Contribuiç̧̄o ao Projeto no 237 ("Gondwana Floras") do Programa Internacional de Correlaçẫo Geológica, IUGS-UNESCO.

2 Trabalho realizado com o apoio financeiro da FAPESP (Proc. № 83/2220-0).

3 Pós-Graduanda do IG-USP e Bolsista da FAPESP (Proc. No 82/2096-5).

4 IG-USP. 
a conchostráceos (ROHN \& RÖSLER, 1985), bilvaves (ROHN, no prelo), estromatólitos nodulares (ROHN \& FAIRCHILD, 1986), Glossopteris (ROHN et al., 1984), Sphenophyllum (RÖSLER \& ROHN, 1984), caules de esfenótitas (ROHN \& RÖSLER, 1986a) e pteridófilas pecopteróides (ROHN \& RÖSLER, 1986b).

\section{MATERIAL E PROCEDÊNCIA}

Os exemplares de $S$. gondwanensis ocorrem na forma de impressōes, sem cutícula e sem quaisquer outros restos orgânicos. Em alguns espécimens, as estruturas aparecem destacadas por contraste cromático. Na maioria dos afloramentos, os fósseis de cada nivel estão mais ou menos concentrados, parcialmente sobrepostos e, aparentemente, representam vegetais depositados em estado fragmentário, nฮึo havendo muitos exemplares com superfícies grandes expostas. Tal problema é frequentemente agravado pela dificuldade em se partir as rochas sedimentares segundo planos homogêneos. Tratamse normalmente de argilitos e argilitos siltosos, cor-de-vinho ou róseos, mais ou menos compactos, com fratura conchoidal. Os fósseis também ocorrem preservados em siltitos e arenitos mujto finos, cinza, roxos ou cor-de-vinho, incipientemente rítmicos com laminaçāo gradacional, favorecendo a partição segundo planos paralelos e, assim, a exposição de porçōes maiores dos fósseis; entretanto, as impressōes nestas litologias apresentam menor resolução ao nivel dos detalhes.

A lista abaixo reúne os principais dados sobre a localização dos afloramentos, litologias dos níveis com $S$. gondwanensis, seu estado de preservação e outros fósseis encontrados nos mesmos niveis. As características litoestratigráficas mais completas dos afloramentos, assim como uma discussāo geral sobre a Formação Rio do Rasto, serão apresentadas em outros trabalhos. Os afloramentos foram numerados segundo as normas do Departamento de Paleontologia e Estratigrafia do Instituto de Geociências da Universidade de São Paulo (sigla AF/ GP). As amostras contendo os $51^{\circ}$ exemplares estudados estăo depositadas na coleçâo paleobotânica do mesmo departamento (sigla GP/3T), sob os números 1621 a 1651,1757 e 1758 .

\section{RELAÇÃO DAS LOCALIDADES}

- AF/GP 168 - estrada Rondinha-Paulo Frontin, a cerca de $400 \mathrm{~m}$ da BR 476, PR; niveis $1,3,4$ e 5 ; argilitos cor-de-vinho; exemplares de $S$. gondwanensis bastante fragmentados, porém com detalhes bem preservados; nivel 1 -
GP/3T $1621 \mathrm{a} / \mathrm{bA}, \mathrm{cB}, 1622 \mathrm{a} / \mathrm{bA}, \mathrm{cB}, \mathrm{a} / \mathrm{cC}$, $\mathrm{b} / \mathrm{cF}, 1623 \mathrm{~A}, 1624 \mathrm{~A}, 1625 \mathrm{~A}, \mathrm{~B}, 1626 \mathrm{~A}$, 1627A, 1628aA; nivel 3 - GP/3T 1629; nivel 4 - GP/3T 1630aA; nivel 5 - exemplares despre. zados. Outros fósseis: Paracalamites sp., Glossopteris sp., folhas e caules indeterminados.

-AF/GP 171 - BR 373, km 310,5, PR; argilito cinza-esverdeado; fragmentos muito pequenos de cf. S. gondwanensis; GP/3T 1757A. Outros fósseis: Paracalamites sp., Glossopteris sp.

- AF/GP 176 - estrada Reserva-Cândido de Abreu, a cerca de $59,8 \mathrm{~km}$ de Reserva. PR; argilito cor-de-vinho; exemplares de cf. $S$. gondwanensis muito mal preservados; GP/3T 1758A, B, C. Outros fósseis: Panacalamites sp.

- AF/GP 179 - BR 476, aproximada. mente km 207,2, PR; argilito cor-de-vinho; frag. mentos muito pequenos de $S$. gondwanensis; $\mathrm{GP} / 3 \mathrm{~T} 1631 \mathrm{a} / \mathrm{bA}$. Outros fósseis: Paracalamites sp., Pecopteris sp., caules indeterminados.

- AF/GP 181 - estrada entre Paula Freitas e a BR 476 , a cerca de $2,3 \mathrm{~km}$ a noroeste da estação ferroviária de Paula Freitas, PR; argilito cor-de-vinho; fragmento pequeno e mal preservado de $S$. gondwanensis; GP/3T $1632 \mathrm{cD}$. Outros fósseis: Paracalamites sp., caules indeterminados.

- AF/GP 182 - BR 476, km 221,2, PR; argilito cor-de-vinho; exemplares de $S$. gondwanensis com detalhes bem preservados, mas muito fragmentados; GP $/ 3 \mathrm{~T} 1633 \mathrm{bB}, 1634 \mathrm{bD}, \mathrm{bE}$. Outros fósseis: Paracalamites sp., caules indeterminados, conchostráceos.

- AF/GP 183 - estrada Poço Preto-Porto Uniảo, a cerca de $200 \mathrm{~m}$ a leste do rio Timbó, SC; MENDES (1954) registrou, neste afloramento, a presença de diversas espécies de conchostráceos; argilito siltoso a arenito fino corde-vinho; exemplares de $S$ gondwanensis não muito bem preservados, em geral, fragmentados; GP/3T 1635aA, 1636aA, b/cB, 1637aA, 1638A. Outros fósseis: Paracalamites sp., cf. Glossopteris sp., cf. Pecopteris, caules, folhas indeterminadas, conchostráceos.

AF/GP 185 - estrada Rondinha-Paulo Frontin, a cerca de $16,8 \mathrm{~km}$ a sul de Paulo Frontin, PR; nível 2; argilito siltoso al terado esverdeado rítmico; um exemplar de $S$. gondwanensis com preservaçāo satisfatória; GP/3T 1639aA. Outros fóssseis: Pecopteris cf. P. dolianitii, Paracalamites sp.

- AF/GP 196 - BR 373, km 102,5, PR; siltito cinza; fragmento pequeno de $S$. gondwanensis; GP/3T 1640. Outros fosseis: Glossopteris sp., $\mathrm{cf}$. Sphenophyllum $\mathrm{sp}$.

- AF/GP 205 - estrada Reserva-Cândido de Abreu, a cerca de 41,1 a 41,3 km de Reserva, 
PR; argilito siltoso róseo; exemplares de $S$. gondwanensis menos fragmentados do que a média e preservação dos detalhes relativamente boa; GP/3T 1641A, 1642A, 1643A, 1644bI, $1645 \mathrm{~A}, 1646 \mathrm{~A}, 1647 \mathrm{aA}, \mathrm{a} / \mathrm{bB}, \mathrm{a} / \mathrm{bC}, \mathrm{a} / \mathrm{bD}$. Outros fósseis: Paracalamites sp., caules indeterminados, conchostráceos.

- AF/GP 210 - BR 376 (Rod. do Café). km 264,6, PR; nivel 2; siltito cinza; exemplares de $S$ gondwanensis muito fragmentados e deformados; GP/3T 1648B. Outros fósseis: Pecopteris dolianitii, Paracalamites sp., caules e folhas inde terminados.

- AF/GP 217 - BR 376, km 273,4, PR: argilito cor-de-vinho; exemplares de $S$ gondwanensis fragmentados: GP/3T $1651 \mathrm{bB}$. Outros fósseis: bivalves Palaeomutela? platinensis.

- AF/GP 227 - PR-90 (Rod. Sapopema-Sāo Jerônimo da Serra), aproximadamente km 118,8, PR; siltito roxo; exemplares de $S$. gondwanensis muito pouco destacados em rela- çăo à matriz, contudo, número de conjuntos foliares em nós sucessivos relativamente alto; GP/ 3T $1649 \mathrm{aA}, \mathrm{aB}, \mathrm{aC}, \mathrm{aD}, \mathrm{bE}, \mathrm{bF}, 1650 \mathrm{bA}, \mathrm{a} / \mathrm{bB}$. aF, 1652B. Outros fósseis: Paracalamites sp.. Pecopteris esperancensis, conchostráceos, bivalves Palaeomutela? platinensis.

\section{SISTEMÁTICA}

\section{Divisão SPHENOPHYTA \\ Classe SPHENOPSIDA \\ Ordem EQUISETALES}

Familia SCHIZONEURACEAE

Gênero Schizoneura Schimper \&

Mougeot, 1844

Schizoneura gondwanensis Feistmantel, 1876

Figs. 1-17

Descrição - Os megafósseis vegetais sāo caracterizados por conjuntos foliares de folhas

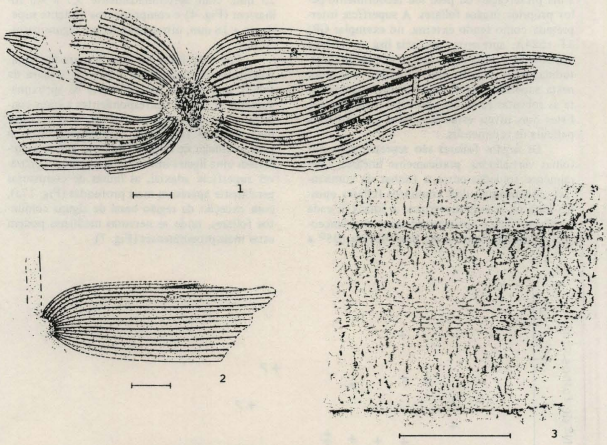

Figuras 1-3 - Schizoneura gondwanensis Feistmantel. Fig. 1 - Dois conjuntos foliares opostos oval-lanceolados com laceração ao longo de algumas linhas de comissuras; espécime GP/3T 1622aA; afioramento AF/GP 168; escala grafica $=5 \mathrm{~mm}$. Fig. 2 - Conjunto foliar ovaloblongo; especime GP/3T 1646A; afloramento AF/GP 205: escala gráfica $=5 \mathrm{~mm}$. Fig. 3 - Detalhe de um conjunto foliar: nervura mediana de uma folha entre linhas de comis: suras; estruturas transversais provavelmente como expressß̈o do mesofilo follar; espécime GP/3T 1621aA; afloramento $A F / G P 168$, escala grafica $=1 \mathrm{~mm}$. 
lineares soldadas entre si, atados a nós de estrestos caules articulados.

Os caules, em geral, estão fragmentados e precariamente preservados, mesmo quando co. nectados a órgăos foliares com boa preservação. Normalmente, há nenhum ou apenas um entrenó completo; tem-se 4 ou 5 entrenós consecutivos somente num exemplar (GP/3T 1649). A largura dos moldes comprimidos dos caules varia entre 3,0 e $4,6 \mathrm{~mm}$ e o comprimento dos entrenós, entre 12,5 e $30,0 \mathrm{~mm}$. As porçōes expostas dos caules correspondem a prováveis moldes da cavidade da medula, e em alguns casos, possivelmente a impressōes da superfície externa. Nos supostos moldes da cavidade da medula são visíveis 7 a 13 estreitas linhas longitudinais, interpretadas como equivalentes aos feixes vasculares, separadas por bandas mais largas, representando as áreas interfasciculares (ou raios medulares primários; vide ROHN \& RÓS. LER, 1986a). O comportamento destas estru. turas não é observável na regiāo dos nós devido à má preservaçāo ou pelo seu recobrimento pe. los próprios órgãos foliares. A superfície interpretada como sendo externa, no exemplar GP/ 3T $1624 \mathrm{~A}$, apresenta aparência lisa, ocorrendo somente impressóes de células alongadas longitudinalmente, muito mal definidas. Contudo, nesta superficie ainda transparecem tenuamente as robustas estruturas do nivel mais interno. Estes dois niveis estão separados por finíssima película de sedimentos.

Os órgđ̃os foliares são representados por folhas verticiladas, praticamente lineares, lateralmente soldadas entre si (linhas de comissuras), constituindo dois conjuntos foliares opostos mais ou menos simétricos. A forma de cada conjunto foliar é aproximadamente oval-lanceolada a oval-oblonga, com base obtusa $\left(95^{\circ}\right.$ a $140^{\circ}$ ), levemente amplexicaule (Figs. 7, 13), e com regiāo distal tendendo a aguda. Os ápices nāo sāo perfeitamente verificáveis por geralmente ocorrerem incompletos ou lacerados ao longo das linhas de comissuras (Fig. 15). Esta laceraçāo também é observada em outras porçōes dos conjuntos foliares, como na base (Fig. 11), na porção média (Fig. 14) ou ao longo de todo o seu comprimento (Figs. 1, 7, 15, 16). Ocorrem 5 a 17 folhas em cada conjunto foliar, geralmente 8 a $13(73 \%)$, havendo número igual ou bastante próximo de folhas nos dois conjuntos foliares opostos. Apesar da má preservação dos caules, verifica-se uma aparente relaçāo (aproximadamente $1: 1$ ) entre o numero de folhas por conjunto foliar e o número de feixes vasculares dos lados expostos dos caules. O número muito baixo de folhas em alguns conjuntos foliares, provavelmente, deve-se ao desprendimento total de algumas folhas devido a rupturas nas comissuras antes da sua deposiçâo. Os conjuntos foliares apresentam larguras entre 6 e $25 \mathrm{~mm}$, com aproximadamente 5,7 a 9,5 folhas/cm (Fig. 4) e comprimentos bastante superiores a $18 \mathrm{~mm}$, ultrapassando, em alguns casos, $55 \mathrm{~mm}$.

Em cada folha há uma nervura mediana, relativamente larga ( $15 \%$ a $30 \%$ da largura da folha), constituida por um feixe de aproximadamente 5 estrias, correspondentes a vasos condutores. As linhas de comissuras e as nervuras medianas sāo facilmente distinguiveis entre si pela diferença de largura. Embora ambas manifestem uma ligeira inflexão para baixo na provável superficie adaxial, as linhas de comissuras geralmente aparecem mais profundas (Fig. 17a), com exceção da região basal de alguns conjuntos foliares, onde as nervuras medianas podem estar mais proeminentes (Fig. 7).

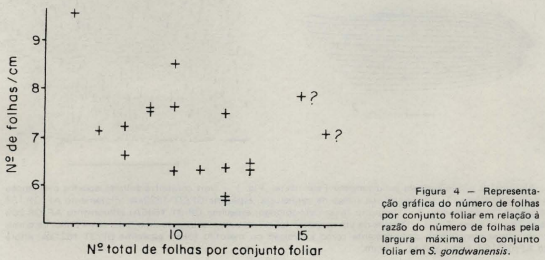


Em vários exemplares, as impressōes dos limbos foliares são caracterizadas por finas estruturas transversais às nervuras medianas $\mathrm{e}$ às linhas de comissuras, com aspecto anastomosado ou apenas sinuoso a reto (Figs. 3, 8, 9, 12a). Estas estruturas são reconheciveis ao observar os fósseis com luz incidente quase paralela à sua superfície e paralela às nervuras. Ainda săo visi. veis outras linhas, muito finas, dispostas longi. tudinalmente nas folhas, raramente nitidas e muito pouco destacadas, perceptiveis com a fonte de luz orientada perpendicularmente a elas.

\section{COMPARAÇŌES E DISCUSSĀO}

Os espécimes analisados assemelham-se, macroscopicamente, à maioria dos exemplares de $S$. gondwanensis já descritos e figurados. Particularmente naqueles exemplares cujos conjun. tos foliares tendem à forma oval-lanceolada (Figs. 1. 7. 12b), o modo de inserção ao caule, a gama de variaçāo das dimensōes, as características das nervuras medianas das folhas e das linhas de comissuras, assim como a laceração dos conjuntos foliares ao longo das linhas de comissuras, sâo aspectos muito semelhantes aos dos exemplares cuidadosamente representados e descritos em FEISTMANTEL (1880). Pode-se citar, como única diferença, o número de folhas por conjunto foliar, aqui variando entre 5 e 17 , enquanto que FEISTMANTEL (1880) observou os extremos de 5 e 14. Schizoneura paradoxa Schimper \& Mougeot, 1844 apresenta número geralmente inferior a 8 folhas por conjunto foliar (FEISTMANTEL, 1880), assemelhando-se, quanto a este aspecto, a alguns dos exemplares examinados. Todavia, $S$. paradoxa difere de $S$. gondwanensis em relaçăo à forma (FEISTMAN. TEL, 1880; BOUREAU, 1964) e ainda, confor-
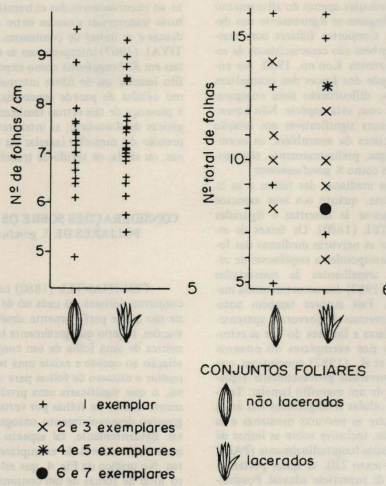

Figura 5 - Representaçåo gráfica do estado de laceraç̋o dos conjuntos foliares nes linhas de comissuras em relaçăo à razão do número de folhas pela largura máxima dos conjuntos foliares em $S$. gonơwanensis. Figura 6 - Representaç̧o gráfica do estado de laceraçăo dos conjuntos foliares nas linhas de comissuras em relaçajo ao número de folhas por conjunto foliar em $S$. gondwanensis. 
me mencionado anteriormente, alguns casos de exemplares com número reduzido de folhas por conjunto foliar podem ser devidos ao desprendimento completo de algumas folhas em consequêencia da laceração.

Os exemplares analisados também são semelhantes aos figurados por MAHESHWARI \& PRAKASH (1965), SURANGE (1966), CAZ. ZULO-KLEPZIG (1978), CAZZULO-KLEPZIG \& CORREIA (1981), CHANDRA \& PRASAD (1981), PANT et al., (1982) e ANDERSON \& ANDERSON (1985). Entretanto, com exceção de PANT et al., (1982) e ANDERSON \& ANDERSON (1985), todos os autores consideraram os conjuntos foliares simplesmente como folhas plurinervadas, sem qualquer referência às linhas de comissuras. O exemplar do Subgrupo Guatá analisado por RIGBY (1972) está muito mal preservado. Provavelmente năo se trata do gênero Schizoneura.

Os conjuntos foliares oval-oblongos (Figs. $2,10,17 \mathrm{~b})$, procedentes apenas do afloramento AF/GP 205, distinguem-se ligeiramente dos demais pela forma. Conjuntos foliares com margens paralelas também são característicos da espécie $S$ manchuriensis Kon'no, 1960. No entanto, não se dispōe dos ápices dos exemplares aqui examinados, dificultando uma comparaçăo mais efetiva com esta espécie. Não havendo outras diferenças significativas em relaçāo aos demais espécimes da assembléia, os exemplares oval-oblongos, preliminarmente, sāo também considerados como $S$. gondwanensis.

As nervuras medianas das folhas e as linhas de comissuras, quanto aos seus aspectos gerais, assemelham-se às descritas e figuradas por FEISTMANTEL (1880). Os feixes de estrias constituindo as nervuras medianas das folhas poderiam corresponder a sequências de cé. lulas alongadas semelhantes às constatadas por PANT et al. (1982) em amostras ainda melhor preservadas. Tais autores também notaram bandas transversais às nervuras, aparentemente mais regulares e lineares do que as estruturas transversais nos exemplares do presente trabalho. PANT et al. (1982) concluiram que estas-bandas transversais provavelmente representam os restos de um mesofilo lamelar. Tam. bém observaram células retangulóides nas áreas intermediárias entre as nervuras medianas e as margens das folhas, inclusive sobre as linhas de comissuras, alinhadas longitudinalmente (PANT et al., 1982, fig.-texto 2B), as quais pertenceriam à epiderme da superfície adaxial. Possivelmente, nos exemplares do presente estudo, as finissimas linhas paralelas às nervuras medianas podem ser impressōes mal preservadas de célu. las epidérmicas alinhadas. Nas áreas entre as nervuras das superficies abaxiais, PANT et al. (1982) verificaram a presença de células mais poligonais (e possiveis estômatos), similares às descritas em SURANGE (1966) para ambas as superficies dos conjuntos foliares. Os exemplares do presente trabalho não estāo suficientemente preservados para confirmar a ocorrência de tais células.

PANT et al. (1982) mencionaram que todas as mesmas características "microscópicas" também são observáveis em Raniganjia bengalensis (Feistmantel) Rigby emend. Pant \& Nautiyal. Macroscopicamente, tal espécie é igualmente similar pela presença de folhas alongadas com uma nervura mediana, soldadas basalmente entre si nas "linhas de comissuras", apenas diferindo por formarem uma única bainha foliar e não dois conjuntos foliares distintos. Entretanto, conforme a descrição e ilustraçōes de $R$. bengalensis apresentadas em PANT \& NAUTIYAL (1967) ainda há diferença quanto ao encurvamento das extremidades das estruturas transversais situadas entre as nervuras medianas e as linhas de comissuras. PANT \&NAUTIYAL (1967) interpretaram as estrias transversais em $R$. bengalensis como expressāo do mesofilo lamelar ou de fibras internas, ou ainda co. mo células de parede espessada. Mencionaram a presença de tais estrias também em formas angáricas de Annularia, aí interpretadas como ex. pressăo do mesofilo lamelar ou de fibras internas, ou ainda, de tecido de transfusão.

\section{CONSIDERACCOESS SOBRE OS CONJUNTOS FOLIARES DE $S$, gondwanensis}

FEISTMANTEL (1880) constatou que os conjuntos foliares em cada nó de $S$. gondwanensis 'são quase perfeitamente simétricos; nas ilustraçð̄es, sugeriu que geralmente há diferença numérica de uma folha de um conjunto foliar em relaçăo ao oposto e existe uma tendência em di. minuir o número de folhas para o ápice do vegetal, o que significaria uma produção gradativamente menor de folhas por verticilo com o progressivo desenvolvimento ontogenético do vegetal. Evidentemente, tal aspecto poderia ser demonstrado através de exemplares mais completos. No gráfico da Fig. 4, que relaciona o número total de folhas de um conjunto foliar e o número de folhas/cm ao longo de uma linha transversal à porçăo mais larga dos conjuntos foliares, verifica-se uma relativa dispersāo dos pontos. Portanto, nāo é possível confirmar que os 
conjuntos foliares menores têm número mais baixo de folhas ou folhas de menor largura. Desta maneira, o gráfico não permite discriminar matematicamente os conjuntos foliares basais ("adultos"), dos apicais ("jovens"), Ou o número de dados considerado não é suficiente, ou a variação observada é devida a outros fatores (ecológicos e/ou tafonômicos).

FEISTMANTEL (1880) também observou a partiçâo dos conjuntos foliares ao longo das linhas de comissuras. No presente trabalho, este caráter ("laceração") foi constatado principalmente nos exemplares do afloramento $\mathrm{AF}$ / GP 168, sugerindo a dependència de fatores locais. Testou-se através de gráficos a possibilidade deste caráter também estar relacionado ao estágio de desenvolvimento ontogenético, indicado, ao menos subjetivamente, pelo número de folhas por conjunto foliar (Fig. 6) ou pela razāo do número de folhas/ $\mathrm{cm}$ ao longo de uma linha transversal à porção mais larga dos conjuntos foliares (Fig. 5). A dispersão dos pontos nos gráficos parece indicar que a laceraçāo independe de tais parâmetros e que realmente deve estar relacionada apenas a fatores tafonômicos (transporte, ressecamento, etc.) ou a fatores ambientais atuantes na planta viva (vento, umi. dade, etc.).

FEISTMANTEL (1880) sugeriu que todas as folhas poderiam ter estado originalmente ligadas formando uma bainha única quando jovens. SURANGE (1966) citou tal hipótese como verdadeira, porém não apresentou provas concretas a este respeito. PANT et al. (1982) verificaram que mesmo em conjuntos foliares pequenos de $S$. gondwanensis, os quais deveriam representar porçōes mais próximas ao ápice de um ramo, existe uma separação nítida entre eles, o que não descarta a possibilidade de que numa fase ainda anterior estivessem unidos. Embora o presente trabalho não tenha contribuido com provas concretas para a elucidação do desenvolvimento ontogenético de $S$. gondwanensis, a hipótese de que todas as folhas de um mesmo verticilo podem ter estado solda. das entre si, num estágio muito jovem, é apoiada pelas seguintes observaçōes: a aparente facilidade de laceração dos conjuntos foliares ao longo das linhas de comissuras; as bases do tipo amplexicaule dos conjuntos foliares; o número de folhas frequientemente diferente entre dois conjuntos foliares de um mesmo verticilo; e a semelhança surpreendente entre Schizoneu$r a$ e Raniganjia quanto à morfologia foliar (o último gênero caracterizado por bainha foliar).

ASAMA (1969) discutiu a ocorrência de vegetais fósseis semelhantes em provincias florísticas separadas, procurando explicar todos os registros desta natureza por evoluçāo paralela dos vegetais. Considerou que $S$. gondwanensis do Gondvana e $S$. manchuriensis da Cathaysia consistem em dois "lobos" formados por fusão de folhas, diferenciando-se apenas pelo caráter da "estriação": em $S$, gondwanensis a estriação indicaria as nervuras medianas de cada folha, enquanto que na outra espécie corresponderia às linhas de sutura entre as folhas. Entretanto, os exemplares de $S$. gondwanensis aqui estudados $\mathrm{e}$ os representados em FEISTMANTEL (1880) e em PANT et al. (1982) também apresentam linhas de comissura pronunciadas $e$, às vezes, sem nervuras medianas muito proeminentes. Desta forma, o caráter de estriação aparentemente não é um critério de distinção adequado em relação à evolução das duas espécies. ASAMA (1969) ainda citou a diferença entre os cones de $S$. gondwanensis e $S$. manchuriensis; entretanto, segundo SURANGE (1966), a estrutura reprodutora atribuida à forma gondvânica por ETHERIDGE (1893, apud SURANGE, 1966), na realidade, deve corresponder a frutificação de glossopterídea. Portanto, a teoria da evolução paralela baseada nos caracteres acima, ao menos na Cathaysia e no Gondvana, deve ser revista.

\section{CONCLUSÕES}

Todos os exemplares estudados no presente trabalho apresentam as caracteristicas diagnosticadas de $S$ gondwanensis que tem ampla distribuição em toda a região gondvânica. A semelhança com as formas descritas por outros autores (FEISTMANTEL, 1880; BOUREAU, 1964; PANT et al., 1982) chega ao nivel de detalhes: as linhas de comissuras das fothas soldadas entre si, as características das nervuras medianas de cada folha, os aspectos relativos provavelmente à epiderme e ao mesofilo foliar. Os conjuntos foliares são oval-lanceolados ou oval-oblongos; os exemplares do segundo tipo sāo procedentes de um único afloramento, na estrada Reserva-Cândido de Abreu. Os dados disponiveis não justificam discriminaçōes baseadas na diferença de forma dos conjuntos foliares.

O fenòmeno da laceração dos conjuntos foliares, também observado em exemplares de outras regióes gondvãnicas, é aqui interpretado como um efeito ambiental.

A ocorrência de $S$. gondwanensis em 13 novas localidades da Formação Rio do Rasto amplia significativamente a extensão geográfica da distribuiçăo da espécie no Brasil, pois havia sido anteriormente registrada em apenas 2 localidades da mesma formaçāo. Na Bacia do Para- 
ná, $S$. gondwanensis restringe-se à Formaçăo Rio do Rasto, podendo ser considerada como mais um elemento da Tafoflora $\mathbf{E}$ de RÖSLER (1978).

\section{AGRADECIMENTOS}

Os autores agradecem ao Dr. H.D. Schors- cher (IG-USP) e ao J. de S. Taddeo (DMGA. IPT) por terem facilitado a utilização de lupas fotográficas das suas respectivas instituiçőes, ao A.G. Rohn pela colaboraçđ̃o nos serviços de fotografia, à S. Czajkowski e à E. Mendonça pelo auxilio nos trabalhos de campo, e ao Dr. T.R. Fairchild pela correçāo do "abstract" e pelas sugestōes apresentadas.

\section{REFERÊNCIAS BIBLIOGRÁFICAS}

ANDERSON, J.M. \& ANDERSON, H.M. (1985) Palaeoflora of southern Africa. Prodromus of South African megafloras, Devonian to Lower Cretaceous. Bot. Research Inst. Rotterdam. A.A. Balkema. 150 p.

ASAMA, K. (1969) Parallelism in Palaeozoic plants between Gondwanaland and Cathaysia Land. IUGS Symposium, Buenos Aires (1967). Gondwana Stratigraphy. Patis, p. 127-136

BOUREAU, E. (1964) Traité de paléobotanique. Tome 3 - Sphenophyta, Noeggerathiophyta. Paris, Masson et Cie. $544 \mathrm{p}$.

CAZZULO-KLEPZIG, M. (1978) Estudo da tafoflórula do Membro Morro Pelado na sua localidade-tipo, Pesquisas, $11: 225-303$

CAZZULO-KLEPZIG, M. \& CORREIA, N. dos R. (1981) Contribuição ao estudo da tafoflora permiana no Membro Serrinha na. Serra do Cadeado, Estado do Paraná, Brasil. II Congr. Latinoam. Paleontol, Porto Alegre, RS. Anais, 1:233-247

CHANDRA, S. \& PRASAD, M.N.V. (1981) Fossil plants from the Kamthi Formation of Maharashtra and their biostratigraphic significance. Paleobot., 28-29:99-121.

ETHERIDGE JR., R. (1893) On the occurrence of a plant allied to Schizoneura in the Hawkesbury Sandstone. Rec. Geol. Surv. New South Wales, 3.

FEISTMANTEL, O. (1880) Flora of the Gondwana System II. The flora of the Damuda and Panchet Divisions (1st Part). Paleontologia Indica, ser. XII, 3(2):1-77.

MAHESHWARI, H.K. \& PRAKASH, G. (1965) Studies in the Glossopteris Flora of India. 21. Plant megafossils from the Lower Gondwana exposures along Bausloi River in Rajmahal Hills, Bihar. Palaeobot., 13(2):115-128.

MENDES, J.C. (1954) Conchostráceos permianos do sul do Brasil. In: LANGE, F.W., Paleontologia do Paraná. Vol. Comem. 19 Centen. Est. Paraná, Curitiba, p. 153-164.

PANT, D.D., MISRA, L.; NAUTIYAL, D.D. (1982) On the structure of stems and leaves of Schizoneura gondwanensis Feistmantel. Palieontograph., Abt. B, 183 (1-3):1-7.

PANT, D.D. \& NAUTIYAL, D.D. (1967) On the structure of Raniganjia bengalensis (Feistm.) Rigby with a discussion of its affinities. Palaeontograph., Abt. B, $121(1-3): 52-64$.

RIGBY, J.F. (1972) The Upper Palaeozoic flora at Lauro Müller, Santa Catarina, Southern Brazil. An. Acad. brasil. Ciênc., 44 (Suplem.): 279-293.

ROHN, R. (no prelo) Bivalves da Formação Rio do Rasto (Permiano Superior) no Estado do Paraná. IX Congr. Bras. Paleontol., fortaleza, CE. Anais.

ROHN, R. \& FAIRCHILD, T.R. (1986) Estromatólitos permianos em calcário coquinóide do Grupo Passa Dois, nordeste do Paraná. An. Acad. brasil. Ciênc., 58:435-446.

ROHN, R.; OLIVEIRA-BABINSKI, M.E.C.B. de; RÖSLER, O. (1984) Glossopteris da Formação Rio do Rasto no sul do Estado do Paraná. XXXIII Congr. Bras. Geol, Rio de Janeiro, RJ. Anais, 2:1047-1061.

ROHN, R. \& RÖSLER, O. (1985) Conchostráceos da Formação Rio do Rasto no sul do Estado do Paraná. VIII Congr. Bras. Paleontol, Rio de Janeiro. MME-DNPM, sér. Geol, 27:481-490 (Paleontol/Estratigr. 2).

ROHN, R. \& RÖSLER, O. (1986a) Caules de Sphenophyta da Formação Rio do Rasto (Bacia do Paraná, Permiano Superior). Neste volume.

ROHN, R. \& RÖSLER, O. (1986b) Pteridófilas pecopteróides da Formação Rjo do Rasto no Estado do Paraná e da Formação Estrada Nova no Estado de Sāo Paulo (Bacia do Paraná, Permiano Superior). Neste volume.

RÖSLER, O. (1978) The Brazilian Eogondvanic floral succession. Bol. IG-USP, 9:85-91.

RÖSLER, O. \& ROHN, R. (1984) Sphenophyllum paranaense n. sp. (Sphenophyta) da Formação Rio do Rasto (Permiano Superior) de Dorizon, Estado do Paraná, Bol. IG-USP, 15:97-104.

SURANGE, K.R. (1966) Indian fossil pteridophytes. Bot. Monogr., New Delhi, Council of Scientific \& Industrial Research, 4:1-209. 